\title{
Preparing ophthalmologists for the use of mechanical ventilation during the COVID-19 pandemic
}

\author{
Joshua P. Harvey $\mathbb{1}^{1} \cdot$ Vita F. Sinclair ${ }^{2}$ \\ Received: 17 April 2020 / Revised: 28 April 2020 / Accepted: 28 April 2020 / Published online: 13 May 2020 \\ (C) The Royal College of Ophthalmologists 2020
}

\section{To the Editor:}

Many ophthalmologists, such as the corresponding author, have already, or will soon find themselves deployed elsewhere, including the intensive care unit (ICU), where mechanical ventilation is central to the care of COVID-19 patients. We propose that an understanding of cataract surgery and phacodynamics well prepares ophthalmologists to understand the principles of mechanical ventilation.

The cataract surgeon is well accustomed for balancing inflow and outflow of fluid to optimise the surgical environment. Similarly during ventilation the inflow and outflow of air must be tightly regulated. Additional considerations are required in acute respiratory distress syndrome (ARDS), a rapid and widespread inflammatory lung disorder, which in a Wuhan population arose in $41.8 \%$ of patients hospitalised with COVID-19 and carried a mortality of 52\% [1].

Ventilation can be defined by the pressures and volumes it generates. The peak inspiratory pressure (PIP), is the maximal pressure obtained at the end of inspiration, Fig. 1. To initiate expiration, the ventilatory pressure drops to a minimal pressure, positive end-expiratory pressure (PEEP), often set at $\sim 5 \mathrm{cmH}_{2} \mathrm{O}$, Fig. 1. The frequency with which the ventilator cycles between these two pressures in $1 \mathrm{~min}$ defines the respiratory rate (RR). This multiplied by the volume generated each cycle gives the minute volume (MV).

The primary role of the infusion in phacoemulsification is to maintain a stable anterior chamber preventing its collapse. The PEEP therefore provides the same function as

Joshua P. Harvey

joshua.harvey@doctors.org.uk

1 Ophthalmology Department, St Thomas' Hospital, London SE1 7EH, UK

2 Anaesthetics Department, St Helier Hospital, Sutton SM5 1AA, UK bottle height by preventing alveolar collapse. In ARDS the lungs stiffen increasing alveolar collapse and ventilation/ perfusion mismatch. Think of these patients like our own challenging (collapsible) patients, the hypermetropic eyes with a narrow anterior chamber. Unfortunately, cohesive viscoelastic is not an option in the lung so to keep the alveoli open in the setting of ARDS, the PEEP is increased, often to $>10 \mathrm{cmH}_{2} \mathrm{O}$, to promote alveolar recruitment and to improve oxygenation [2].

During phacoemulsification we can alter the contents of the infusion fluid to optimise our surgery. Similarly by increasing the fraction of inspired oxygen ranging from 0.21 $(21 \%)$ or room air to $1(100 \%)$, we can improve oxygenation.

In the most complex of cataract cases, if the lens nucleus drops, sometimes we require a vitreoretinal surgeon to approach things from a different angle. In the ventilation of patients with ARDS, gravity will cause the localised collapse of posterior segments of the lung whilst simultaneously shunting blood to these poor oxygenated portions of the lung, increasing V/Q mismatch. Placing patients in

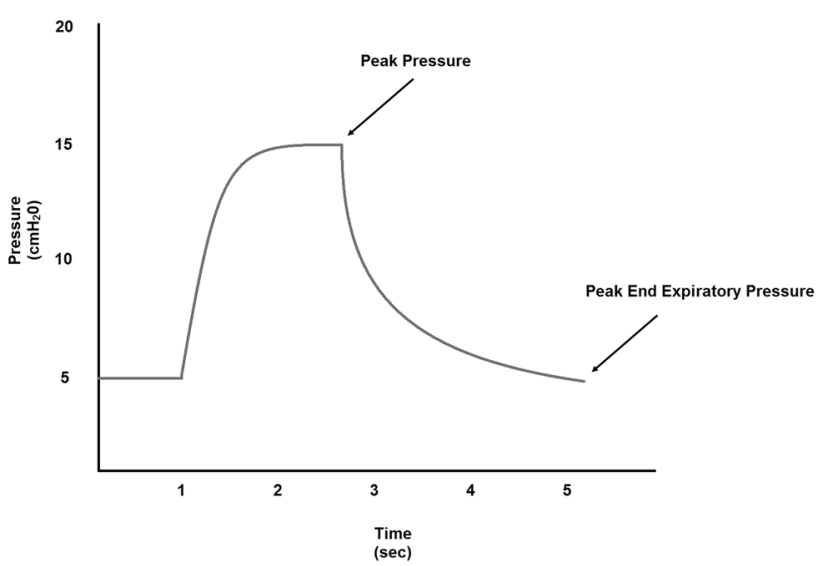

Fig. 1 Graph demonstrating airway pressure as a function of time over a single respiratory cycle. Airway pressure increases to peak pressure during inspiration before falling to peak end expirartory pressure at the end of expiration. 
Table 1 Demonstration of the key differences between volume and pressure control.

\begin{tabular}{llll}
\hline Mode of ventilation & Independent variable & Dependent variable & Advantage \\
\hline Volume control & Vt, PEEP, RR & PIP & Controls tidal volume to protect lung from hyper-inflation/volume trauma \\
Pressure control & PIP, PEEP, RR & Tidal volume & Controls maximal pressure to protect lung from barotrauma \\
\hline
\end{tabular}

$V t$ tidal volume, $P E E P$ positive end-expiratory pressure, $R R$ respiratory rate, $P I P$ peak inspiratory pressure.

the prone position is a technique to counter gravity dependant collapse/atelectasis and as for post macular hole surgery patients, longer is better and 16+ hours is advised [3].

Together the Fi02, PEEP and prone positioning are three tools for addressing hypoxia. Prone positioning is increasingly used in treating patients with COVID-19 and therefore, it is likely, our technical skills will be called upon to aid in the complex manoeuvre of placing patients in prone position in the ICU.

Central to understanding phaco fluidics is an understanding of what pump system we are using. A peristaltic system adjusts the speed of the rotation of the peristaltic rollers to maintain the desired vacuum but a venturi system will use an inflow/outflow differential of pressurised gas. These two systems can help us understand ventilator control modes.

The two main ways of driving flow to the lung are pressure control and volume control (Table 1). In volume control, the ventilator delivers a constant tidal volume $(\mathrm{Vt})$ and the ventilator titrates the pressure (PIP) in accordance with the patient's thoracic compliance.

In pressure control the clinician defines the ventilatory pressures and the $\mathrm{Vt}$ becomes the dependent variable. In the context of COVID-19 related lung disease, pressure control may be preferable because the operator is able to limit barotrauma by setting a ceiling pressure on the ventilator. Lung damage is reduced at the expense of lower tidal volumes, $\mathrm{MV}$ and therefore reduced $\mathrm{CO}_{2}$ clearance. Conversely, to increase $\mathrm{CO}_{2}$ clearance, either the RR or $\mathrm{Vt}$ must be increased, however the ARDS.net study showed that smaller tidal volumes, $6-8 \mathrm{ml} / \mathrm{kg}$ IBW, improve outcomes despite a higher $\mathrm{CO}_{2}$ [4].

In summary, ophthalmologists can use the principles outlined here, under the guidance of our ICU colleagues to understand basic ventilator parameters, modes and common issues.

\section{Compliance with ethical standards}

Conflict of interest The authors declare that they have no conflict of interest.

Publisher's note Springer Nature remains neutral with regard to jurisdictional claims in published maps and institutional affiliations.

\section{References}

1. Wu C, Chen X, Cai Y, Xia J, Zhou X, Xu S, et al. Risk factors associated with acute respiratory distress syndrome and death in patients with coronavirus disease 2019 pneumonia in Wuhan, China. JAMA Intern Med. 2020. Published online.

2. Guo L, Xie J, Huang Y, Pan C, Yang Y, Qui H, et al. Higher PEEP improves outcomes in ARDS patients with clinically objective positive oxygenation response to PEEP: a systematic review and meta-analysis. BMC Anesthesiol. 2018;18:172.

3. Guérin C, Reignier J, Richard JC, Beuret P, Gacouin MD, Boulain $\mathrm{T}$, et al. Prone positioning in severe acute respiratory distress syndrome. N Engl J Med. 2013;368:2159-68.

4. Network TA. Ventilation with lower tidal volumes as compared with traditional tidal volumes for acute lung injury and the acute respiratory distress syndrome. The Acute Respiratory Distress Syndrome Network. N Engl J Med. 2000;342:1301-8. 